\title{
Dynamique Socio-économique de L'adoption de la Transformation Améliorée de la Farine Fermentée du Manioc en République Démocratique du Congo (RDC)
}

\author{
Moloba Lukombo Yannick,
}

Ingénieur Agroéconomiste et Assistant de Recherche

MULTINA-DMK, Bureau d'études de l'Université de Kinshasa, Faculté des Sciences Agronomiques, Université de Kinshasa, RDC

Mobula Meta Victor, (PhD, Professeur)

Ntoto Mvubu Roger, (PhD, Professeur)

Mpanzu Balomba Patience, (PhD, Professeur)

Belani Massamba Justin, (MSc, Chef des Travaux)

Ngonde Nsakala Hervé, Assistant de Recherche

Département d'Economie Agricole,

Faculté des Sciences Agronomiques de l'Université de Kinshasa, RDC

Mahungu Nzola Meso, (PhD, Professeur)

Lukumbo Singi Simon, MSc

Associé de Recherche, Institut International d'Agriculture Tropicale, RDC Botumba Kupesa Toussaint, Me

Assistant de Recherche Institut Supérieur d'Études Agronomiques (ISEA), RDC

\section{Doi:10.19044/esj.2019.v15n27p187 URL:http://dx.doi.org/10.19044/esj.2019.v15n27p187}

\section{Résumé}

La transformation du manioc demeure artisanale, avec souvent une faible performance sur la qualité du produit. Pour la promouvoir, la diffusion des innovations agricoles va continuer à progresser suivant le contexte socioéconomique. Globalement, l'étude vise à analyser le processus d'adoption de la technologie des micro-cossettes. Spécifiquement, elle vise à déterminer les facteurs socio-économiques qui l'influencent. L'enquête a été conduite à l'aide du questionnaire individuel, administré à 112 transformateurs répartis dans les provinces de Kongo Central et de la Tshopo. Face aux coûts élevés de cette innovation agricole, l'approche de dotation des kits de transformation, en faveur des Organisations Paysannes, est préconisée. Ainsi, les services sont gratuitement offerts aux membres. Cependant, $57 \%$ d'entre-eux y recourent, 
pour produire le Fufu (farine fermentée). La meilleure qualité du produit final, la facilité de l'obtenir et le séchage rapide des micro-cossettes sont les principales motivations. La femme est toujours active dans cette activité postrécolte moderne. Malgré cette gratuité des services, la concurrence déloyale sur le marché et les charges logistiques constituent les obstacles, selon $43 \%$ des non-adoptants. L'adoption est positivement influencée par la main d'œuvre externe, l'état des routes d'accès aux marchés ruraux et urbains (Matadi et Kisangani), alors qu'elle est négativement influencée par l'éloignement aux stations de recherche (Gimbi et Yangambi). L'émergence de cette activité moderne, stimulera l'offre en matière première. Elle pourrait être considérablement appuyée par des facteurs socio-économiques. Il s'agit de la main d'œuvre, des infrastructures routières d'accès aux marchés (ruraux et urbains) et l'accès à l'information technologique.

Mots-clés: Adoption, Technologie, Innovations agricoles 


\title{
Socio-Economic Dynamics of the Adoption of Improved Processing Cassava Fermented Flour in Democratic Republic of the Congo (DRC)
}

\author{
Moloba Lukombo Yannick, \\ Ingénieur Agroéconomiste et Assistant de Recherche \\ MULTINA-DMK, Bureau d'études de l'Université de Kinshasa, \\ Faculté des Sciences Agronomiques, Université de Kinshasa, RDC \\ Mobula Meta Victor, (PhD, Professeur) \\ Ntoto Mvubu Roger, (PhD, Professeur) \\ Mpanzu Balomba Patience, (PhD, Professeur) \\ Belani Massamba Justin, (MSc, Chef des Travaux) \\ Ngonde Nsakala Hervé, Assistant de Recherche \\ Département d'Economie Agricole, \\ Faculté des Sciences Agronomiques de l'Université de Kinshasa, RDC \\ Mahungu Nzola Meso, (PhD, Professeur) \\ Lukumbo Singi Simon, MSc \\ Associé de Recherche, Institut International d'Agriculture Tropicale, RDC \\ Botumba Kupesa Toussaint, Me \\ Assistant de Recherche Institut Supérieur d'Études Agronomiques (ISEA), \\ RDC
}

\begin{abstract}
Cassava processing remains artisanal, often with poor performance on product quality. In a bid to promote it, there need to be a progression in agricultural innovations according to socioeconomic context. This paper, however, focuses on analyzing the adoption process of microchip technology. Specifically, it aims to determinate the socio-economic factors that influence it. A survey was conducted using individual questionnaire, which was administrated to 112 processors in provinces of Kongo Central and Tshopo. In view of the high costs of this agricultural innovation, the dotation approach of processing kits, in favor of farmer's organizations, was promoted. Services were offered free of charge for members. 57\% of the members employ them to produce Fufu (fermented flour). Best quality of final product, ease of producing it, and quick drying of microchip constitute the main motivations. Women were majorly active in the modern post-harvest activity. Despite the
\end{abstract}


fact the services were offered free of charge, unfair competition in the market and logistic costs were the main obstacles according to $43 \%$ of non-adopters. Adoption is positively influenced by external labor and state of access roads to rural and urban markets (Matadi and Kisangani). On the other hand, it is negatively influenced by remoteness to research stations (Gimbi and Yangambi). Emergence of that modern activity will stimulate raw material supply, and it should be significantly supported by socioeconomic factors. These include external labor, roads infrastructures of access to markets (rural and urban), and access to technological information.

Keywords: Adoption, Technology, Agricultural innovations

\section{Introduction}

Le manioc (Manihot esculenta Crantz) est l'aliment de base de la population de la République Démocratique du Congo (RDC). La consommation moyenne annuelle par habitant, est estimée à $450 \mathrm{~kg}$ de racines fraîches (Khonde, 2001 ; Chausse et al., 2012 ; Ndonda, 2018). Cette quantité correspond à $150 \mathrm{~kg}$ de farine fermentée. En plus, les feuilles de manioc sont consommées comme légumes. D'ailleurs, Ndonda (2018) a estimé que la consommation moyenne annuelle par congolais est de $29 \mathrm{~kg}$ de ces feuilles. Chausse et al. (2012) estiment que la consommation annuelle du manioc par habitant, tous produits confondus, est d'environ $250 \mathrm{~kg}$. Cette consommation est parmi la plus élevée au monde. Vers les années 1990, la production annuelle des racines tubéreuses du manioc a baissé de $24 \%$ (FAO, 2009). Des vastes programmes urgents de lutte contre les maladies et ravageurs, l'ont stabilisé de 14,93 millions de tonnes en 2002 à 15,02 millions de tonnes en 2011 (MINAGRIDER, 2013). La mise au point et la distribution de nouvelles variétés améliorées visent à augmenter la production du manioc, avec leur accès facile par les paysans (Kouassi et al., 2018).

Après la récolte, la détérioration physiologique des racines tubéreuses du manioc est accélérée (Koko et al., 2014). Par conséquent, elles peuvent être consommées directement, ou servir de matières premières pour une multitude de produits de valeur ajoutée élevée, grâce à la transformation (Nweke et al., 2000 ; James et al., 2013). Les méthodes traditionnelles de transformation sont rudimentaires et fastidieuses, aboutissant souvent aux produits transformés de qualité relativement instable. Elles ne permettent pas toujours de maintenir la couleur blanche des macro-cosettes (produits semi-finis), première qualité exigée sur le marché de gros et détail, surtout pendant le séchage naturel (quatre à sept jours). Ensuite, les macro-cossettes sont transformées en Fufu, farine fermentée qui constitue le produit fini.

En effet, le séchage naturel constitue souvent un goulet d'étranglement, surtout dans une zone tropicale où il pleut abondamment. 
Après séchage, la teneur en eau du produit sec doit être suffisamment réduite, car c'est un paramètre très important du stockage d'un produit sec. La teneur en eau idéale se situe entre 8 et 10 \% (Kwatia \& Jeon, 1990 ; IITA, 2014). Pour les macro-cossettes, la qualité de la couleur est souvent inférieure suite à une forte teneur en eau, qui accroît la vulnérabilité aux moisissures. La compétitivité de la filière exige non seulement des progrès importants au niveau de la productivité des variétés améliorées, mais à tous les niveaux de la chaîne des valeurs (James et al., 2013 ; Contech et al., 2015). Les besoins de transformation du manioc en Fufu (farine fermentée) de meilleure qualité (couleur blanche, surtout) constituent toujours un réel défi.

En dépit de la stabilisation de la production nationale du manioc depuis 2002, Nweke et al. (2000) mentionnent que la transformation n'a pas été stimulée au rythme de cette production, comme il en a été le cas en Afrique de l'Est. Pour réduire davantage le temps de séchage naturel et la teneur en eau, le recours à la technologie des micro-cossettes est l'une des solutions préconisées en milieu paysan. En plus, la transformation artisanale rudimentaire et fastidieuse accuse des limites, face à de quantités importantes de la matière première à traiter. Ceci est l'une des faiblesses de la performance de cette activité post-récolte.

La diffusion de la technologie des micro-cossettes, qualifiée d'innovation agricole, prend de plus en plus de l'ampleur depuis 2004. Produits transformés semi-finis substituables aux macro-cossettes, elles constituent la matière première du Fufu. Cette technologie s'opère avec des kits de transformation, constitués le plus souvent d'un découpeur, d'une râpedécoupeuse et d'une presse, par moment d'un moulin et d'un bac de rouissage. Elle permet de produire un Fufu plus compétitif, et stimuler l'offre de la matière première auprès des paysans.

L'approvisionnement en matière première est principalement assuré par la propre production, car le transformateur est avant tout producteur (Akoroda, 2007). En outre, les kits appuient la transformation, comme le signifie Osswald (1995) que la proportion de manioc transformé augmente de 56 à $91 \%$ dans les villages, qui possèdent ne fût-ce qu'une râpe. Khonde (2001) et Rusike et al. (2012) considèrent que l'augmentation de la productivité technologique et production du manioc, sont significativement importantes, pour assurer la sécurité alimentaire et accélérer la croissance économique, afin de réduire la pauvreté.

Depuis l'introduction des micro-cossettes, la demande de cette technologie ne cesse de croître jusqu'à ce jour. Cependant, elle est très coûteuse, en termes d'acquisition des kits de transformation. Ces kits sont très coûteux (plus de 1.500 USD), à tel point que les paysans sont incapables de se les procurer par leur agriculture de subsistance. Ainsi, l'approche de dotation des kits, en faveur des Organisations Paysannes (OP), pour une utilisation 
communautaire, est préconisée (IITA, 2014). Les partenaires techniques et financiers utilisant cette approche, subventionnent les coûts élevés d'acquisition. Par contre, seuls les membres des organisations bénéficiaires, accèdent gratuitement aux services offerts.

En effet, les études initiées sur le manioc sont nombreuses et diversifiées. Elles ont mis un accent très fort sur l'amélioration variétale, l'accroissement de rendement, la transformation, la commercialisation et la consommation. Les études relatives au processus d'adoption technologique sur la transformation du manioc, suivant une chronologie des paramètres significatifs, sont encore très peu réalisées. Les données demeurent éparpillées sans être centralisées jusqu'à ce jour. Et pourtant, ces études focalisées sont très pertinentes. Elles orientent les décisions politiques, susceptibles d'améliorer les programmes d'interventions sur cette filière.

L'objectif général de cette étude est d'analyser le processus d'adoption de la technologie des micro-cossettes, dans l'option de la transformation améliorée du Fufu. Elle vise spécifiquement à déterminer les facteurs socioéconomiques, susceptibles d'accélérer efficacement les attitudes d'adoption, vis-à-vis de cette innovation agricole. Dans le cadre actuel d'appuis à la chaîne des valeurs manioc, l'hypothèse de l'étude stipule que la diffusion de la technologie des micro-cossettes connaît un franc succès. Ce succès est mesuré par rapport à l'adoption auprès des paysans, qui progresse suivant le contexte socio-économique.

\section{Approches méthodologiques}

\section{a. Milieu d'étude}

L'étude s'est réalisée dans les provinces de Kongo Central et la Tshopo, telles qu'illustrées sur la figure 1. Elles sont appuyées par le projet de recherche agricole pour le développement des cultures stratégiques en Afrique (SARD-SC : Support to Agricultural Research for Development of Strategic Crops), exécuté par IITA. 


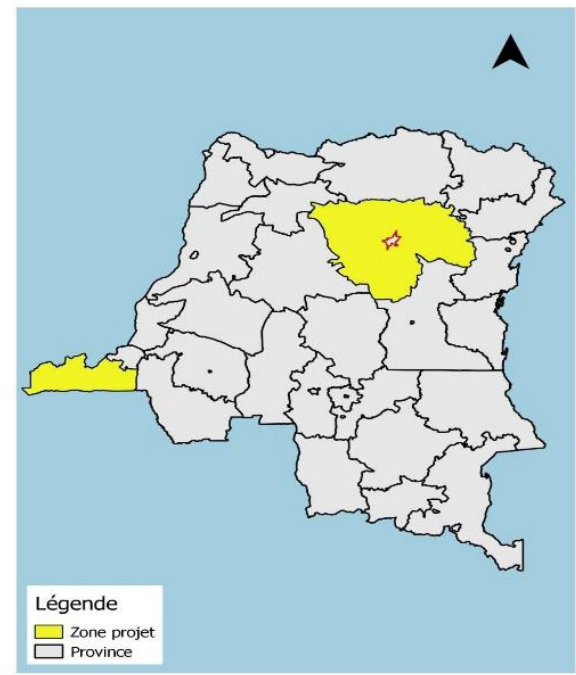

Figure 4: Subdivision administrative de la RDC et province sous étude

L'unique province qui donne accès à l'Océan Atlantique, la province de Kongo Central $\left(53.947 \mathrm{~km}^{2}\right)$ possède Matadi ( $05^{\circ} 49^{\prime}$ 03" Sud) comme ville principale. Elle abrite une diversité de paysages constitués des forêts denses et humides, forêts résiduelles, savanes herbeuses et mangroves sur la côte. Son climat tropical humide est marqué par les saisons pluvieuse et sèche. Ce climat est influencé par le courant marin froid de Benguela sur la région littorale. À l'instar de l'économie nationale, celle de cette province est dominée par le secteur agricole. Elle abrite la station de Gimbi $\left(05^{\circ} 31^{\prime}\right.$ Sud et $13^{\circ} 32^{\prime}$ Est $)$ qui y conduit les activités de recherche sur le manioc dans la région de Mayombe. L'étude a été réalisée autour de cette station, à Seke-Banza et Moanda, qui sont les deux (2) territoires administratifs ciblés.

La province de la Tshopo (199.567 $\left.\mathrm{km}^{2}\right)$ avec Kisangani $\left(0^{\circ} 31^{\prime} 09^{\prime \prime}\right.$ Nord et $25^{\circ} 11^{\prime} 46^{\prime \prime}$ Est) comme ville principale, est située dans les plaines avec une forêt primaire et de nombreuses zones inondables. Son climat est de type tropical chaud et humide sans saison sèche marquée. Son économie est basée sur l'agriculture de subsistance et l'élevage traditionnel. Elle abrite la station de recherche de Yangambi $\left(0^{\circ} 46^{\prime} 03^{\prime \prime}\right.$ Nord et $24^{\circ} 26^{\prime} 29^{\prime \prime}$ Est), la plus grande De l'Institut National d'Etude et Recherche Agronomiques (INERA). L'étude a été réalisée autour de cette station, au niveau des territoires de Banalia et Isangi.

\section{b. Collecte des données}

Différentes sources documentaires ont été consultées et passées en revue, pour appréhender le phénomène complexe du processus d'adoption. L'étude a employé la démarche analytique basée sur l'approche objet. 
L'approche est centrée sur les caractéristiques de l'innovation considérée individuellement (OECD, 2005). Elle suggère que soit dressée une liste des innovations, en se conviant souvent sur les évaluations d'experts. La réduction du temps de séchage et celle de la teneur en eau sont les caractéristiques de l'innovation. La collecte des données primaires a été effectuée à l'aide du questionnaire administré aux transformateurs, et la grille d'entretiens semistructurés adressée aux informateurs-clés, en s'appuyant sur la synergie des partenaires locaux.

A l'issue de la pré-enquête ayant permis le repérage des kits de transformation et le test du questionnaire, l'étude a exploité les données recueillies en mai 2016. L'enquête in situ par sondage aléatoire stratifié, a été conduite auprès de 112 transformateurs du manioc (60 au Kongo Central et 52 à la Tshopo), membres des OPs bénéficiaires des kits de transformation. La liste des membres a servi de référence pour les identifier, selon la disponibilité à être interviewé. A l'absence des listes actualisées des membres, seule la disponibilité a été considérée. Cependant, la classification des acteurs identifiés est inclusive, c'est-à-dire un acteur est considéré producteur et transformateur du manioc. Cette taille représente une grande taille à la distribution normale des paramètres, au regard du théorème central-limite.

Les différents sites en référence aux conditions socio-écologiques, ont été stratifiés en zones rurales et urbaines. Seuls les transformateurs des zones rurales, membres des OPs, ont été ciblés et sélectionnés de manière aléatoire. L'étude a été réalisée sur un rayon de $163 \mathrm{~km}$, autour de stations de recherche de Gimbi (Kongo Central) et Yangambi (Tshopo).

\section{c. Analyses des données}

Le recours aux statistiques descriptives s'est avéré indispensable. Les tendances centrales et de dispersion ont été ressorties sur certaines variables quantitatives pour mieux appréhender leurs caractéristiques statistiques. En effet, deux groupes d'acteurs ont été créés, à savoir les adoptants et nonadoptants. Les données sont analysées au sein de ces groupes, afin d'évaluer la tendance des variables dans la perspective d'adoption de l'innovation.

En outre, la régression logistique, à travers le modèle économétrique Probit, a été appliquée. Le modèle du choix binaire (ou encore appelé modèle dichotomique) est utilisé, dès que la variable dépendant ne peut prendre que deux modalités ( 0 ou 1 ). Cependant, les signes de coefficients de ce modèle indiquent si la variable agit positivement ou négativement sur la probabilité $\mathrm{P}$ value (Wooldridge, 2000).

L'effet marginal des variables significatives a été dégagé, en vue de déterminer la probabilité de leur influence. Pour l'équation de la régression, le modèle suivant a été utilisé : $\mathrm{y}=\beta_{0}+\beta_{1} \mathrm{x}_{1}+\beta_{2} \mathrm{x}_{2}+\beta_{3} \mathrm{x}_{3}+\ldots+\beta_{\mathrm{n}} \mathrm{x}_{\mathrm{n}}+\varepsilon$, où $\mathrm{y}$ : 
variable dépendante binaire (Adoption), $\mathrm{x}_{1}$ à $\mathrm{x}_{\mathrm{n}}$ : variables indépendantes, $\beta_{0}$ à $\beta_{\mathrm{n}}$ : paramètres à estimer et $\varepsilon$ : terme d'erreur.

\section{d. des variables explicatives}

Le modèle économétrique a intégré les variables explicatives, en référence aux théories socio-économiques et études similaires, qui ont développé la réflexion sur le processus d'adoption des innovations. Les variables explicatives choisies sont : le genre du chef de ménage, son état civil (statut marital), son âge, son niveau d'instruction, la taille du ménage, le recours à la main d'œuvre externe, l'état de routes d'accès aux marchés ruraux et urbains ainsi que la distance des sites par rapport aux marchés ruraux, urbains et la station de recherche. Donc, l'adoption est un processus socioéconomique dynamique évoluant au rythme de sa diffusion.

\section{Résultats et discussion}

Les données sont présentées suivant une analyse de la tendance des variables dans la perspective d'adoption de la technologie des micro-cossettes, afin de tenter de comprendre leur différence au sein des groupes d'adoptants et non-adoptants. Le Tableau 1 présente les caractéristiques socioéconomiques des producteurs enquêtés.

Tableau 1 : Caractéristiques socio-économiques des ménages enquêtés

\begin{tabular}{|c|c|c|c|c|c|c|}
\hline \multirow{2}{*}{ Variables (unité) } & \multirow{2}{*}{ Modalité } & \multicolumn{2}{|c|}{ Kongo Central } & \multicolumn{2}{|c|}{ Tshopo } & \multirow{2}{*}{ Total } \\
\hline & & $\begin{array}{l}\text { Seke- } \\
\text { Banza }\end{array}$ & Moanda & Banalia & Isangi & \\
\hline \multirow{2}{*}{ Genre $(\%)$} & Femme & 28,0 & 17,1 & 0,0 & 0,0 & 11,6 \\
\hline & Homme & 72,0 & 82,9 & 100,0 & 100,0 & 88,4 \\
\hline \multirow{6}{*}{ Age (Ans) } & Moyenne & 47,9 & 44,6 & 42,6 & 43,3 & 44,6 \\
\hline & Minimum & 29,0 & 23,0 & 27,0 & 23,0 & 23,0 \\
\hline & Maximum & 71,0 & 67,0 & 65,0 & 67,0 & 71,0 \\
\hline & Ecart-type & 11,7 & 11,1 & 12,7 & 11,5 & 11,6 \\
\hline & $\mathrm{CV}$ & 0,2 & 0,2 & 0,3 & 0,3 & 0,3 \\
\hline & Variance & 136,7 & 124,1 & 160,3 & 131,3 & 135,1 \\
\hline \multirow{4}{*}{ Etat civil (\%) } & Célibataire & 8,0 & 11,4 & 0,0 & 0,0 & 5,4 \\
\hline & Marié & 72,0 & 77,1 & 94,7 & 93,9 & 83,9 \\
\hline & Veuf (ve) & 12,0 & 5,7 & 0,0 & 3,0 & 5,4 \\
\hline & Divorcé (e) & 8,0 & 5,7 & 5,3 & 3,0 & 5,4 \\
\hline \multirow{3}{*}{$\begin{array}{l}\text { Niveau d'instruction } \\
(\%)\end{array}$} & Sans instruction & 12,0 & 11,4 & 0,0 & 0,0 & 6,3 \\
\hline & Primaire & 28,0 & 37,1 & 15,8 & 21,2 & 26,8 \\
\hline & Secondaire & 36,0 & 48,6 & 84,2 & 78,8 & 60,7 \\
\hline
\end{tabular}




\begin{tabular}{llccccc} 
& Supérieur/Universitaire & 24,0 & 2,9 & 0,0 & 0,0 & 6,3 \\
\hline & Moyenne & 6,6 & 5,0 & 9,2 & 9,3 & 7,3 \\
& Minimum & 1,0 & 1,0 & 3,0 & 3,0 & 1,0 \\
Taille du ménage & Maximum & 12,0 & 10,0 & 24,0 & 23,0 & 24,0 \\
(Personne) & Ecart-type & 3,3 & 2,1 & 4,7 & 4,7 & 4,2 \\
& CV & 0,5 & 0,4 & 0,5 & 0,5 & 0,6 \\
& Variance & 11,2 & 4,3 & 22,5 & 22,5 & 17,5 \\
\hline \multirow{2}{*}{ Adoption (\%) } & Non-adoptant & 24,0 & 80,0 & 68,4 & 3,0 & 42,9 \\
& Adoptant & 76,0 & 20,0 & 31,6 & 97,0 & 57,1 \\
\hline \multirow{2}{*}{ Main d'œuvre } & Non & 28,0 & 62,9 & 26,3 & 9,1 & 33,0 \\
extérieure (\%) & Oui & 72,0 & 37,1 & 73,7 & 90,9 & 67,0 \\
\hline
\end{tabular}

Source : Données de l'enquête, 2016

Le Tableau 1 révèle que le genre masculin est plus dominant, et seulement $12 \%$ des ménages sont dirigés par une femme. Néanmoins, la femme demeure toujours active dans cette transformation améliorée, comme dans la transformation artisanale. Toutefois, l'homme semble intéressé de plus en plus à cette transformation semi-industrielle, car elle mobilise une main d'œuvre importante en termes de logistique. Bien que certaines opérations sont confiées à l'homme, les deux genres participent à cette activité postrécolte. D'ailleurs, Kinkela et al. (2007) ont constaté la répartition des tâches entre les deux genres dans la filière manioc. Il s'observe une différence significative entre les adoptants et non-adoptants. Le genre du chef de ménage pourrait avoir une influence positive dans l'attitude de l'adoption de la technologie.

Les enquêtés sont des adultes avec un âge moyen de 45 ans, et il est noté une hétérogénéité de l'âge. La majorité d'entre eux sont mariés et instruits (Tableau 1). La taille moyenne du ménage est de sept (7) personnes par rapport au Tableau 1, et il y a une forte variation dans la composition. Ce qui présume une disponibilité des actifs dans ces familles nombreuses. Au sujet de l'âge, état civil, instruction et taille du ménage, il n'est signalé aucune différence entre les adoptants et non-adoptants. Donc, ces variables n'auraient aucune influence dans la probabilité d'adoption.

Les institutions très actives dans la dotation des kits de transformations en faveur des OPs, pour promouvoir la technologie des micro-cosettes, demeurent l'IITA, la FAO (Organisation des Nations Unies pour l'Alimentation et l'Agriculture) et l'Enabel (Agence Belge de Développement, ex-Coopération Technique Belge : CTB). Plus de la moitié des enquêtés (57 \%) recourent gratuitement aux services des kits de transformation, et produisent principalement le Fufu (farine fermentée). La meilleure qualité du produit final, la facilité de l'obtenir et le séchage rapide 
des micro-cossettes sont les principales motivations. Par ailleurs, le Kimpuka (pâte rouie) et la farine panifiable (farine non fermentée) sont aussi des produits transformés lors de l'usage des kits de transformation.

Malgré la gratuité des services, les membres non-adoptant (43\%) ont évoqué les raisons majeures suivantes : la concurrence déloyale sur le marché entre les produits obtenus par la transformation améliorée et la transformation traditionnelle et les charges logistiques diverses (transport, etc.). La majorité des adoptants (89\%) emploient une main d'œuvre externe, pour acheminer les racines tubéreuses aux kits de transformation, et les éplucher. En plus, il se dégage une différence significative entre les deux groupes (adoptants et nonadoptants) au sujet de la main d'œuvre externe. Elle pourrait influencer positivement l'adoption.

Les routes qui relient les villages aux marchés ruraux et urbains sont en bon état, car les véhicules peuvent y rouler à une vitesse moyenne de plus de $45 \mathrm{~km} / \mathrm{h}$. Les sites d'enquête sont généralement accessibles. D'ailleurs, l'état des routes d'accès aux marchés urbains (Matadi et Kisangani) est positivement lié à l'adoption. Donc, l'adoption pourrait être positivement influencée par l'état des routes d'accès aux marchés urbains.

L'adoption de cette technologie ne dépend pas seulement de ses avantages, mais c'est un processus socio-économique actif impliquant plusieurs facteurs pour être localement adopté. Les variables retenues dans le modèle sont spécifiées au Tableau 2 ci-dessous.

Tableau 2 : Spécification des variables du modèle économétrique Probit

\begin{tabular}{|c|c|c|c|c|c|c|c|}
\hline $\begin{array}{l}\text { Variables } \\
(\mathrm{N}=112)\end{array}$ & Unité & Modalité & Moy & $\begin{array}{l}\text { Ecart- } \\
\text { type }\end{array}$ & Min & Max & $\begin{array}{l}\text { Effet } \\
\text { espéré }\end{array}$ \\
\hline Adoption & & & 0,6 & 0,5 & 0,0 & 1,0 & \\
\hline Genre (GR) & & $\begin{array}{l}0=\text { Fém } \\
1=\text { Masc }\end{array}$ & 0,9 & 0,3 & 0,0 & 1,0 & + \\
\hline Etat civil (EC) & & $\begin{array}{l}0=\text { Autres } \\
1=\text { Marié }\end{array}$ & 0,8 & 0,4 & 0,0 & 1,0 & $?$ \\
\hline Age $(\mathrm{AG})$ & Ans & & 44,6 & 11,6 & 23,0 & 71,0 & - \\
\hline Instruction (IN) & & $\begin{array}{l}0=\text { Aucune } \\
1=\text { Instruction }\end{array}$ & 0,9 & 0,2 & 0,0 & 1,0 & + \\
\hline Taille ménage (TM) & Personne & & 7,3 & 4,2 & 1,0 & 24,0 & + \\
\hline $\begin{array}{l}\text { Main d'œuvre } \\
\text { extérieure (MO) }\end{array}$ & & $\begin{array}{l}0=\text { Non } \\
1=\text { Oui }\end{array}$ & 0,7 & 0,5 & 0,0 & 1,0 & + \\
\hline $\begin{array}{l}\text { Etat de la route du } \\
\text { marché rural (EM) }\end{array}$ & $\mathrm{Km} / \mathrm{h}$ & & 47,6 & 11,7 & 12,5 & 60,2 & + \\
\hline $\begin{array}{l}\text { Etat de la route du } \\
\text { marché urbain }(\mathrm{EV})\end{array}$ & $\mathrm{Km} / \mathrm{h}$ & & 45,8 & 14,3 & 28,3 & 75,9 & + \\
\hline $\begin{array}{l}\text { Distance du marché } \\
\text { rural (DM) }\end{array}$ & $\mathrm{Km}$ & & 2,3 & 3,7 & 0,4 & 16,7 & - \\
\hline
\end{tabular}


Distance du marché urbain (DV)
$\mathrm{Km}$

$\mathrm{Km}$
88,3

68,1
43,3

52,5
$31,0 \quad 159,9$

\section{Distance de} l'INERA (DI)

Source : Données de l'enquête, 2016

Pour tenter d'apprendre la décision de recourir à la technologie des micro-cossettes, les variables influençant l'adoption sont déterminées au Tableau 3. Le sens de l'influence et l'effet marginal de ces variables significatives y sont ressortis.

Tableau 3 : Estimation des variables de l'adoption et l'effet marginal

\begin{tabular}{|lccccc|}
\hline Adoption & Coef. & Std. Err. & $\mathbf{z}$ & $\mathbf{P}>\mathbf{z}$ & Effet marginal \\
\hline GR & $-0,344$ & 0,826 & $-0,420$ & 0,677 & $-0,060$ \\
EC & 0,885 & 0,678 & 1,300 & 0,192 & 0,155 \\
AG & $-0,001$ & 0,017 & $-0,090$ & 0,931 & 0,000 \\
IN & $-0,825$ & 0,874 & $-0,940$ & 0,345 & $-0,144$ \\
TM & $-0,018$ & 0,051 & $-0,340$ & 0,730 & $-0,003$ \\
MO* & 1,361 & 0,414 & 3,290 & 0,001 & 0,238 \\
EM* & 0,036 & 0,015 & 2,490 & 0,013 & 0,006 \\
EV* & 0,069 & 0,027 & 2,560 & 0,011 & 0,012 \\
DM & $-0,066$ & 0,058 & $-1,130$ & 0,256 & $-0,012$ \\
DV & 0,007 & 0,008 & 0,850 & 0,394 & 0,001 \\
DI* & $-0,024$ & 0,005 & $-5,150$ & 0,000 & $-0,004$ \\
Constante & $-3,706$ & 2,079 & $-1,780$ & 0,075 & \\
\hline
\end{tabular}

Source : Données de l'enquête, 2016. * : variable significative à $\mathrm{p}<0,01{ }^{*}{ }^{*}$ : variable significative à $\mathrm{p}<0,05 . * * *$ : variable significative à $\mathrm{p}<0,1$

La main d'œuvre externe, l'état des routes d'accès aux marchés ruraux et urbains (Matadi et Kisangani) influencent positivement l'adoption de la technologie des micro-cossettes au seuil significatif de $1 \%$. La main d'œuvre externe augmente la probabilité d'adoption de $24 \%$, eu égard à son effet marginal (Tableau 3). La probabilité de l'adoption s'accroît de $1 \%$, suivant les effets marginaux de l'état des routes d'accès aux marchés ruraux et urbains (Tableau 3). Par contre la distance par rapport aux stations de recherche (Gimbi et Yangambi) exerce une influence opposée à l'adoption au seuil significatif de $1 \%$. Son effet marginal diminue la probabilité de l'adoption de $1 \%$.

Plus la quantité de la matière première augmente, plus la main d'œuvre externe est sollicitée. En amont, elle est mobilisée pour emblaver des superficies raisonnables de production des racines tubéreuses, car la plupart des transformateurs sont avant tout producteur de cette matière première. En 
aval, elle est mobilisée pour le transport et l'épluchage de la matière première, qui sont des opérations qui s'effectuent manuellement.

Le secteur de transformation en se modernisant progressivement, est pourvoyeur d'emploi en milieu rural, surtout pour les femmes et jeunes (Hillocks, 2002 ; Soule et al., 2013 ; Contech et al., 2015). L’émergence du secteur engendre celle de plusieurs métiers connexes, dont l'artisanat pour fabriquer des presses et râpeuses, tout en propulsant la demande de ces équipements agricoles. La main d'œuvre externe exerce une influence positive modérée (effet marginal de $24 \%$ ). Néanmoins, Kouakou (2017) confirme que la main d'œuvre a un effet positif et significatif sur l'efficacité technique.

L'amélioration des routes d'accès aux marchés ruraux et urbains favorise l'accès aux débouchés. Par conséquent, elle renforce le rythme de diffusion des innovations agricoles, par la circulation aisée des intrants et produits agricoles (Mastaki, 2006 ; Bonkena, 2014). Les paysans sont sûrs d'écouler leurs produits transformés, soit par la collecte assurée par les commerçants au niveau des marchés ruraux, soit par la vente directe aux marchés urbains (Bolakonga et al., 2013 ; Mpanzu, 2013).

Ils ont une attitude positive, vis-à-vis des choix technologiques, car l'accès aux différents marchés de collecte et/ou de vente directe des produits est toujours garanti. Cette recherche montre que l'influence positive des routes demeure très faible (effets marginaux de $1 \%$ ). Ce constat susciterait des doutes sur l'impact de la seule route par rapport au développement agricole. D’ailleurs, il est aussi révélé par Cook (1998) et Bonkena (2014). Ils ont suggéré que les investissements agricoles complémentaires et l'amélioration du climat macro-économique, s'opèrent simultanément avec l'amélioration du réseau routier, pour engendre plus d'impacts socio-économiques dans les zones rurales.

L'éloignement vis-à-vis des stations de recherche (Gimbi et Yangambi) est une fonction décroissante par rapport à l'adoption technologique. Plus les ménages s'y éloignent, moins ils adoptent la nouvelle technologie. En effet, cet éloignement anéantit les perspectives d'accès à l'information, vis-à-vis des choix technologiques (Mastaki, 2006). Ce fait influence négativement les choix à opérer dans la transformation du manioc, surtout dans un pays où la recherche technologique demeure sous financée, à cause des multiples crises politico-économiques.

Les stations de Gimbi et Yangambi sont dans un état criant de manque des ressources humaines et financières, caractérisé par le ralentissement, le dysfonctionnement ou l'arrêt de plusieurs programmes de recherche. L'absence de motivation a accéléré la fuite des cerveaux, par le départ massif du personnel vers d'autres opportunités jugées meilleures (Tollens, 2016). Bien que désenclavé, la mobilité du personnel est très limitée à Gimbi. En plus, Yangambi se trouve dans un état d'enclavement quasi total. En outre, 
Tollens (2016) relève que la recherche technologique demeure peu performante, surtout au niveau de couverture. Il note trois (3) chercheurs pour 100.000 agriculteurs en RDC. Toutefois, l'éloignement aux stations de recherche réduit faiblement l'adoption technologique (effet marginal de $1 \%$ ).

\section{Conclusion}

L'étude s'est développée autour de l'objectif d'analyser le processus d'adoption de la technologie des micro-cossettes du manioc. Dans le cadre de la relance de l'activité post-récolte, la diffusion de cette innovation technologique coûteuse (plus de 1.500 USD) se fait par l'approche de dotation des kits de transformation. La dotation s'effectue au profit des Organisations Paysannes (OPs), pour une utilisation communautaire, dans l'option d'améliorer davantage la valeur ajoutée. Dans la zone de la présente étude (Kongo Central et Tshopo), l'approche est plus soutenue par l'IITA, la FAO et l'Enabel (ex-Coopération Technique Belge : CTB).

Vis-à-vis des résultats de l'étude, il ressort que $57 \%$ des transformateurs interrogés recourent aux services des kits de transformation. Ils sont gratuitement offerts aux membres des OPs bénéficiaires, qui produisent principalement le Fufu (farine fermentée). Cette technologie est adoptée, à cause de la meilleure qualité du produit final, la facilité de l'obtenir et le séchage rapide de micro-cossettes. Hormis, le Fufu obtenu à partir des micro-cossettes, le Kimpuka (pâte rouie) et la farine panifiable (farine non fermentée) constituent les autres produits transformés. Toutefois, la femme est toujours active dans les tâches de cette activité post-récolte moderne, bien qu'il est aussi signalé une implication effective de l'homme. Par ailleurs, les non-adoptants ( $43 \%$ ) pensent que la concurrence déloyale sur le marché et les charges logistiques diverses liées à cette technologie sont encore des obstacles majeurs.

En effet, l'adoption technologique est positivement influencée par la main d'œuvre externe, l'état des routes d'accès aux marchés ruraux et urbains (Matadi et Kisangani), alors qu'elle est négativement influencée par l'éloignement aux stations de recherche (Gimbi et Yangambi). L'émergence de cette activité post-récolte moderne, pourvoyeuse d'emploi en milieu rural, stimulera aussi l'offre en matière première pour soutenir la compétitive de la chaîne des valeurs manioc. Cette émergence pourrait être considérablement appuyée par des facteurs socio-économiques, pour réduire la pauvreté et soutenir la sécurité alimentaire. Il s'agit principalement de la main d'œuvre, des infrastructures routières d'accès aux marchés (ruraux et urbains) et l'accès à l'information technologique. 


\section{Remerciements}

Nos profondes gratitudes sont exprimées au projet SARD-SC, mis en œuvre par l'IITA sur financement de la Banque Africaine de Développement (BAD), pour les appuis logistiques et financiers.

\section{References :}

1. Akoroda, M.O. (2007). Systèmes visant à assurer l'approvisionnement en racines de transformation de manioc des usines de transformation. In: Amani, G., Nindjin, C., N'zue, B., Tschannen, A. \& Aka, D. (ed), Potential of Cassava (Manihot esculenta Crantz) processing in West Africa. CTA, FARA and AUF, 341 p.

2. Bolakonga, I.A., Disonama, M., Baudouin, M. \& Lebailly, P., (2013). Système de production, économie rurale et accès au marché en milieu Mbole, territoire d'Opala, République Démocratique du Congo. In: Badibanga, T. \& Ulimwenga, J. (ed), Development of agriculture in Democratic Republic of Congo: opportunities and constraints. Paris (France): Harmattan, p. 42-62.

3. Bonkena, B.P. (2014). Infrastructures routières et développement socioéconomique dans le Mayombe (RDC). Harmattan, 181 p.

4. Chausse, J.P., Kembola, T. \& Ngonde, R. (2012). L’agriculture : Pierre angulaire de l'économie de la RDC. In: Herderschee, J., Samba, M.D. \& Tshimenga, T.M. (ed), Résilience d'un Géant Africain : Accélérer la croissance et promouvoir l'emploi en République Démocratique du Congo. Volume II : Etudes sectorielles. Kinshasa, Médiaspaul, p.1-98.

5. Contech, A.M.H, Yan, X. \& Moiwo, J.P. (2015). The determinants of grain storage technology adoption in Sierra Leone. Cahiers Agricultures 24: 47-55. DOI : 10.1684/agr.2015.0733.

6. Cook, C.C. (1998). L'analyse sociologique dans les projets de routes rurales. In Cernea, M.M. (ed), La dimension humaine dans les projets de développement : les variables sociologiques et culturelles, Karthala, 586 p.

7. FAO (2009). Deuxième rapport national sur l'état des ressources phytogénétiques pour l'alimentation et l'agriculture en RDC. Rome, $67 \mathrm{p}$.

8. Hillocks, R.J. (2002). Cassava in Africa. In: Hillocks, R.J., Thresh, J.M. and Belotti, A.C. (ed.), Cassava: Biology, Production and Utilization. London (UK): University of Greenwich, p. 41-54.

9. IITA (2014). Research for development to support the cassava industry in the Democratic Republic of Congo (Cassava III). Final report, p 4750.

10. James, B., Okechukwu, R., Abass, A., Fannah, S., Maziya-Dixon, B., Sanni, L., Osei-Sarfoh, A., Fomba, S. \& Lukombo, S., (2013). 
Production du gari à partir du manioc : Guide illustré à l'intention des transformateurs de manioc à petite échelle. IITA, $32 \mathrm{p}$.

11. Khonde, M. (2001). Transformation, Commercialisation et Consommation du Manioc : une stratégie de sécurité alimentaire à Kinshasa. In: Kankonde, M. \& Tollens, E. (ed), Sécurité alimentaire au Congo-Kinshasa: production, consommation et survie. Paris, Harmattan, p. 173-222.

12. Kinkela, S., Bahandi, A., Ntoto, M. \& Belani, M., (2007). Analyse Genre dans le système de production du manioc : cas de plateau de Bateke à Kinshasa. In Herd, T. \& Okana, N. (ed), Lutte contre la pauvreté en RDC: Bilan et perspective. Faculté Catholique de Kinshasa, p. 85-111.

13. Koko, A.C., Kouame, K.B., Anvoh, B.Y., Amani, N.G. \& Assidjo, N.E. (2014). Comparative study on physicochemical characteristics of cassava roots from three local cultivars in Côte d'Ivoire. European Scientific Journal, Edition Vol. 10, No 33 ISSN: 1857-7881, p. 418432.

14. Kouassi, K.M., Mahyao, A., N’zue, Boni, Koffi, E. \& Koffi, C. (2018). Status of Cassava (Manihot esculenta Crantz) in Côte d'Ivoire: From Production to Consumption and Evaluation of Technology Adoption. European Scientific Journal, Edition Vol. 14, No 9 ISSN: 1857-7881, p. $285-299$.

15. Kouakou Kouakou, P.A. (2017). Analyse de la performance productive de l'agriculture urbaine dans le district d'Abidjan. European Scientific Journal. Edition Vol 13, No 35 ISSN : 1857-7881, p. 288-301.

16. Kwatia, J. \& Jeon, W.Y. (1990). Transformation du manioc. In: IITA (ed), Le manioc en Afrique Tropicale : un manuel de référence. IITA, p 99-123.

17. Mastaki, N.J. (2006). Rôle des goulots d'étranglement de la commercialisation dans l'adoption des innovations agricoles chez les producteurs du Sud-Kivu (Est de la R.D.Congo). Gembloux, Thèse de doctorat, $400 \mathrm{p}$.

18. MINAGRIDER (2013). Annuaire des statistiques 2006-2011. Médiaspaul, Kinshasa, 110p.

19. Mpanzu, B.P. (2013). Filière vivrière du Bas-Congo : Acteurs, fonctionnement et performances. In: Badibanga, T. \& Ulimwengu, J. (ed), Development of agriculture in Democratic Republic of Congo: opportunities and constraints. Harmattan, $138 \mathrm{p}$.

20. Ndonda, M.A. (2018). Evaluation agronomique des champignons mycorhiziens locaux sur la productivité du manioc (Manihot esculenta 
Crantz) en sols dégradés des jachères herbeuses à Kisangani/R.D Congo. Université de Kisangani, Thèse de doctorat, 209 p.

21. Nweke, F., Lutete, A.G.O., Dixon, Ugwu, B., Ajobo, O., Kalombo, N. \& Bukaka, B. (2000). Casava production and processing in the Democratic Republic of Congo, Collaborative Study in Africa (COSCA), Working Paper $n^{\circ} 22$, IITA, Ibadan.

22. OECD (2005). Oslo Manual: Guidelines for collecting and interpreting data, 3rd ed. Organisation for Economic Co-operation and Development (OECD), 163 p. http://www.oecdilibrary.org/docserver/download/9205111e.pdf?expires $=1516378717$ \&id=id\&accname=guest $\&$ checksum $=$ F984323C350D0987FE15E1D C9501A96C

23. Osswald, P. (1995). Economie des racines et tubercules. Solagral,66 p.

24. Rusike, J., Mahungu, N.M., Lukombo, S.S., Kendenga, T., Bidiaka, S.M., Alena, A., Lema, A. \& Manyong, V.M. (2012). The impact of the emergency response to the outbreak of cassava mosaic disease in the Democratic Republic of Congo. In Okechukwu \& Ntawuruhunga (ed), Tropical roots and tuber crops and challenges of globalization and climate change. Proceeding of 11th ISTRC-AB symposium, Kinshasa, Democratic Republic of Congo, 4-8 Octobre 2010.

25. Soule, G.B., Aboudou, F., Gansari, S. Tassou, M. \& Yallou, D.J. (2013). Analyse de la structure et la dynamique de la chaîne des valeurs du manioc au Benin. LARES, $70 \mathrm{p}$.

26. Tollens, E. (2016). Les parcs agro-industriels et l'agriculture familiale : les défis du secteur agricole en RDC. In: Marysse, S. \& Tshonda, J.O. (ed), Conjonctures congolaises 2015 : entre incertitudes politiques et transformation économique. Paris (France) : Harmattan et Tervuren : Musée royal de l'Afrique Centrale, p. 147-158.

27. Wooldridge, M.J. (2000). Introductory Econometrics: A Modern Approach. South-Western College, p. 540-546. 